\title{
Word-of-Mouth Communication and Percolation in Social Networks
}

\author{
Arthur Campbell \\ Online Appendix
}

\section{A Appendix}

\section{Representing Social Networks with Random Graphs with Arbitrary Degree Distributions}

Derivatives The probability $p_{k}$ is given by the $k$ th derivative of $G_{0}$ according to:

$$
p_{k}=\left.\frac{1}{k !} \frac{d^{k} G_{0}}{d x^{k}}\right|_{x=0}
$$

Moments Moments of the probability distribution can be calculated from the derivative of the generating function. The $m$ th moment equals:

$$
\sum_{k} k^{m} p_{k}=\left[\left(x \frac{d}{d x}\right)^{m} G_{0}(x)\right]_{x=1}
$$

Where the average degree, which I denote by $z_{1}$, is given by $z_{1}=G_{0}^{\prime}(1)=\sum_{k} p_{k} k$ and the terminology $\left(x \frac{d}{d x}\right)^{m}$ means repeating $m$ times the operation: differentiate with respect to $x$ and then multiply by $x$.

Powers The distribution of the sum of $m$ independent draws from the probability distribution $\left\{p_{k}\right\}$ is generated by the $m$ th power of the generating function $G_{0}(x)$. For example, if I choose two individuals at random from the population and sum together the number of friends each person has then the distribution of this sum is generated by the function $\left[G_{0}(x)\right]^{2}$. To see this, consider the expansion of $\left[G_{0}(x)\right]^{2}$ :

$$
\begin{aligned}
{\left[G_{0}(x)\right]^{2}=} & {\left[\sum_{k} p_{k} x^{k}\right]^{2} } \\
= & \sum_{j, k} p_{j} p_{k} x^{j+k} \\
= & p_{0} p_{0} x^{0}+\left(p_{0} p_{1}+p_{1} p_{0}\right) x^{1} \\
& +\left(p_{0} p_{2}+p_{1} p_{1}+p_{2} p_{0}\right) x^{2} \\
& +\left(p_{0} p_{3}+p_{1} p_{2}+p_{2} p_{1}+p_{3} p_{0}\right) x^{3} \ldots
\end{aligned}
$$

In this expression the coefficient of the power of $x^{l}$ is the sum of all products $p_{k} p_{j}$ such that $k+j=l$. It is therefore the probability that the sum of the degrees of the two individuals will be $l$. This property can be extended to any power $m$ of the generating function. 


\section{Proof of Result 1}

Proof. Results for the case of independent valuations and connections is given by both Cohen et. al.(2000) and Callaway et. al. (2000) which show via different means that the critical percolation $q_{c}$ threshold satisfies

$$
q_{c}=\frac{1}{G_{1}^{\prime}(1)}
$$

If one sets $q_{c}=1-P^{c r i t}$, the expression is then equivalent to $F^{\prime}\left(1, P^{c r i t}\right)=1$ for the case of independent valuations. Callaway et. al. also cover the case where the percolation probability is correlated with number of degrees where the threshold is defined by $F^{\prime}\left(1, P^{\text {crit }}\right)=1$. In addition, earlier work by Molloy and Reed (1995) develop an expression for the critical transition for a graph without percolation in terms of the degree distribution. The result also requires that $F^{\prime}(1, P)$ is decreasing in $P$. This is immediate by noting that

$$
F^{\prime}(1, P)=\frac{\sum_{k} k p_{k} q_{k}(P)}{\sum_{k} k p_{k}}
$$

and $\frac{d q_{k}(P)}{d P} \leq 0$. Finally $\frac{\partial F^{\prime}(1, P)}{\partial P}<0$ at $F^{\prime}(1, P)=1$ ensures $P^{c r i t}$ is unique.

\section{Proof of Theorem 1}

Proof. Demand is given by equation 14

$$
\begin{aligned}
D(P) & =(1-P) \sum_{k} p_{k}\left(1-H_{1}(1, P)^{k}\right) \\
& =(1-P) \sum_{k} p_{k}\left(1-u(P)^{k}\right)
\end{aligned}
$$

where $u(P)=H_{1}(1, P)$ is the smallest non-negative solution to the self consistency condition:

$$
\begin{aligned}
u(P) & =1-F_{1}(1)+F_{1}(u(P)) \\
& =P+(1-P) G_{1}(u(P))
\end{aligned}
$$

The following lemma gives some properties of $u$ with respect to the price which will be used to prove the theorem.

Lemma 1 Suppose $u(P)$ is given by equation 6 then

1. $u(P)=1$ and $\frac{d u}{d P}=0$ for $P^{\text {crit }} \leq P \leq 1$

2. $u<1$ and $\frac{d u}{d P}>0$ for $0 \leq P<P^{c r i t}$

3. $u(P)$ is continuous in $P$

Proof. $u(P)$ is the smallest non-negative solution to:

$$
u=P+(1-P) \frac{\sum_{k} k p_{k} u^{k-1}}{z_{1}}
$$

Now consider the function $f(u)=P+(1-P) \frac{\sum_{k} k p_{k} u^{k-1}}{z_{1}}$. First, the solution $u=1$ always satisfies the above relationship. Second, $f(u)$ is a polynomial in $u$ with positive coefficients, thus it is continuous, increasing 
and convex in the region $0 \leq u \leq 1$ and, given $f(0)=P$, there is at most one other solution $0 \leq u<1$.

When $f^{\prime}(1) \leq 1$ there is no solution for $0 \leq u<1$; and $u=1$ is the unique solution. When $f^{\prime}(1)>1$ there is a solution for $0 \leq u<1$. The condition $f^{\prime}(1) \leq 1$ is equivalent to $P>P^{\text {crit }}$ :

$$
\begin{gathered}
f^{\prime}(1)=(1-P) \frac{\sum_{k} k(k-1) p_{k}}{z_{1}} \leq 1 \\
1-\frac{z_{1}}{\sum_{k} k(k-1) p_{k}} \leq P \\
P^{c r i t} \leq P
\end{gathered}
$$

Therefore, $u=1$ for $P \geq P^{\text {crit }}$ and $0 \leq u<1$ for $P<P^{\text {crit }}$. Hence, $\frac{d u}{d P}=0$ for $1 \geq P \geq P^{\text {crit }}$. For $P<P^{\text {crit }}$ the derivative $\frac{d u}{d P}$ is:

$$
\frac{d u}{d P}=\frac{\left(1-G_{1}(u)\right)^{2}}{1-G_{1}(u)-(1-u) G_{1}^{\prime}(u)}
$$

The numerator is positive for $u<1$ and the denominator $1-G_{1}(u)-(1-u) G_{1}^{\prime}(u)$ is continuous and equal to 1 at $u=0$, equal to 0 at $u=1$ and is decreasing in $u$ for $0 \leq u \leq 1$ provided $G_{1}^{\prime \prime}(1)>0$. This is a necessary condition for $P^{c r i t}>0$. Hence, in the range $P \in\left[0, P^{c r i t}\right), u(P)$ is continuous and $\frac{d u}{d P}>0$.

Returning to the theorem, I conclude that $D(P)=0$ for $P \in\left(P^{\text {crit }}, 1\right]$ and $D(P)=(1-P)\left(1-\sum_{k} p_{k} u^{k}\right)$ for $P \in\left[0, P^{\text {crit }}\right)$. Also, $D(P)$ is a continuous function from the continuity of $u$ in $P$ over these two intervals. I prove the continuity of $D(P)$ for the entire interval $P \in[0,1]$ by showing that as the price approaches the critical price from below $D \rightarrow 0$. The relationship between $P$ and $u$ may be written:

$$
P(u)=\frac{u-G_{1}(u)}{1-G_{1}(u)}
$$

such that $P(u)$ is a continuous, monotonically increasing (one to one) function $[0,1) \rightarrow[-1,1]$. I will now show that $\lim _{u \rightarrow 1^{-}} P(u)=P^{c r i t} . P(1)=\frac{0}{0}$ so applying L'Hopital's rule

$$
\begin{aligned}
\lim _{u \rightarrow 1^{-}} P(u) & =\lim _{u \rightarrow 1^{-}} P^{\prime}(u) \\
& =\frac{1-G_{1}^{\prime}(1)}{G_{1}^{\prime}(1)} \\
& =1-\frac{E[k]}{E\left[k^{2}\right]-E[k]} \\
& =P^{\text {crit }}
\end{aligned}
$$

Now, since $P(u)$ is a one-to-one function and $0<P^{\text {crit }}<1$, this implies that $\lim _{P \rightarrow P^{c r i t-}-u=1 \text { and hence }}$ $\lim _{P \rightarrow P^{\text {crit }}-} D=0$

This completes the argument for the continuity of $D$. The next part of the theorem is:

For $P<P^{\text {crit }} \frac{d D}{d P}<0$

Proof. Consider the expression for $\frac{d D}{d P}$ :

$$
\frac{d D}{d P}=-\left[1-\sum_{k} p_{k} u^{k}+(1-P) \frac{d u}{d P} \sum_{k} p_{k} k u^{k-1}\right]
$$


The result follows from $u<1$ and $\frac{d u}{d P}>0$ for $P<P^{\text {crit }}$.

The final element of the proof is:

For $P<P^{\text {crit }}\left|\frac{P}{D} \frac{d D}{d P}\right|>\left|\frac{P}{1-P}\right|$

Proof. The expression for the elasticity is:

$$
\frac{P}{D} \frac{d D}{d P}=-\frac{P}{1-P}\left[1+\frac{(1-P)}{1-\sum_{k} p_{k} u^{k}} \frac{d u}{d P} \sum_{k} p_{k} k u^{k-1}\right]
$$

where the second term inside the brackets is strictly positive, from lemma 1 . The result follows immediately.

\section{Proof of Theorem 2}

Proof. Define the fully informed monopoly price as $P_{F I}^{*}$ and the WOM monopoly price as $P_{W O M}^{*}$. A monopolist facing a fully informed population has a strictly concave profit maximization problem and charges the unique monopoly price $P_{F I}^{*}=\frac{1+c}{2}$ provided $c<1$. If $c \geq 1$ then there is clearly no price where the monopolist can make positive profits. It is also true that

$$
\frac{P-c}{P} \geq \frac{1}{\varepsilon_{F I}} \text { for any } P \geq P_{F I}^{*}
$$

Theorem 1 proved that $\left|\varepsilon_{W O M}\right|>\left|\varepsilon_{F I}\right|$, which implies that:

$$
\frac{P-c}{P}>\frac{1}{\varepsilon_{W O M}} \text { for any } P \geq P_{F I}^{*}
$$

when demand is positive in the range of prices $P^{\text {crit }}>P \geq P_{F I}^{*}$. The WOM monopolists profit function $(P-c) D(P)$ is continuous and differentiable for $P<P^{c r i t}$. Therefore the first order conditions for the monopolist are necessary and hence $\frac{P-c}{P}>\frac{1}{\varepsilon_{W O M}}$ for all $P \geq P_{F I}^{*}$ implies $P^{M o n} \ngtr P_{F I}^{*}$.

\section{$5 \quad$ Proof of Theorem 3}

Proof. In a Poisson network:

$$
\begin{aligned}
D(P) & =(1-P)\left(1-\exp \left[z_{1}(u-1)\right]\right)=u \\
\frac{d D}{d P} & =-\frac{d u}{d P}=\frac{1-\exp \left[z_{1}(u-1)\right]}{1-(1-P) z_{1} \exp \left[z_{1}(u-1)\right]} \\
\frac{P}{D} \frac{d D}{d P} & =-\frac{P}{1-P} \frac{1}{1-(1-P) z_{1} \exp \left[z_{1}(u-1)\right]}
\end{aligned}
$$

Hence, the standard mark-up formula for the optimal price gives:

$$
\begin{aligned}
\frac{P^{*}-c}{P^{*}} & =-\frac{1}{\frac{P}{D} \frac{d D}{d P}} \\
& =\frac{1-P^{*}}{P^{*}}\left(1-\left(1-P^{*}\right) z_{1} \exp \left[z_{1}\left(u\left(P^{*}, z_{1}\right)-1\right)\right]\right)
\end{aligned}
$$


This is sufficient for profit maximization provided that:

$$
\begin{aligned}
\frac{\partial\left(\left(1-P^{*}\right) z_{1} \exp \left[z_{1}\left(u\left(P^{*}, z_{1}\right)-1\right)\right]\right)}{\partial P} & >0 \\
-z_{1} \exp \left[z_{1}\left(u\left(P^{*}, z_{1}\right)-1\right)\right]+\left(1-P^{*}\right) z_{1} \frac{1-\exp \left[z_{1}(u-1)\right]}{1-(1-P) z_{1} \exp \left[z_{1}(u-1)\right]} \exp \left[z_{1}\left(u\left(P^{*}, z_{1}\right)-1\right)\right] & >0 \\
\left(1-P^{*}\right) z_{1} \frac{1-\exp \left[z_{1}(u-1)\right]}{1-(1-P) z_{1} \exp \left[z_{1}(u-1)\right]} & >1
\end{aligned}
$$

which is true when $\left(1-P^{*}\right) z_{1}>1$. The condition $z_{1}>1$ is sufficient for this to be the case since $P^{*}<P^{\text {crit }}$ and $1-P^{\text {crit }}=\frac{1}{z_{1}}$. To complete the proof we show

$$
\begin{aligned}
\frac{d\left(z_{1} \exp \left[z_{1}\left(u\left(z_{1}\right)-1\right)\right]\right)}{d z_{1}} & <0 \\
\frac{d\left(z_{1} \exp \left[z_{1}(u-1)\right]\right)}{d z_{1}} & =\exp \left[z_{1}(u-1)\right]+z_{1}(u-1) \exp \left[z_{1}(u-1)\right]+\frac{d u}{d z_{1}}\left(z_{1}\right)^{2} \exp \left[z_{1}(u-1)\right] \\
& =\left(1+z_{1}(u-1)+\frac{d u}{d z_{1}}\left(z_{1}\right)^{2}\right) \exp \left[z_{1}(u-1)\right]
\end{aligned}
$$

where

$$
\begin{aligned}
\frac{d u}{d z_{1}} & =\frac{(u-1)(1-P) \exp \left[z_{1}(u-1)\right]}{1-(1-P) z_{1} \exp \left[z_{1}(u-1)\right]} \\
& =\frac{(u-1)(u-P)}{1-z_{1}(u-P)}
\end{aligned}
$$

substituting this in and dropping $\exp \left[z_{1}(u-1)\right]>0$ from the expression:

$$
\begin{aligned}
1+z_{1}(u-1)+\frac{(u-1)(u-P)}{1-z_{1}(u-P)}\left(z_{1}\right)^{2} & <0 \\
1-z_{1}(1-u)\left[1+\frac{z_{1}(u-P)}{1-z_{1}(u-P)}\right] & <0 \\
1-z_{1}(1-u)\left[\frac{1}{1-z_{1}(u-P)}\right] & <0 \\
1-z_{1}(u-P) & <z_{1}(1-u) \\
1 & <z_{1}(1-P)
\end{aligned}
$$

which is true since $1-P^{\text {crit }}=\frac{1}{z_{1}}$ and for positive demand $P^{*}<P^{\text {crit }}$.

\section{Proof of Theorem 4}

Proof. The appropriate generalization of $P^{\text {crit }}$ for the case of correlation between valuations and friendships is:

$$
P^{\text {crit }}=\sup \left\{P \mid F_{1}^{\prime}(1, P)=1\right\}
$$

A sufficient condition for $P^{\text {crit }}>\underline{\theta}$ is $\sum_{k} \frac{p_{k} q_{k}(\underline{\theta})}{z_{1}} k(k-1)>1$. Suppose that for $k \geq 2$ valuations $\theta$ are uniformly distributed over $[0, c] \cup[\underline{\theta}, 1]$ and no people with $k=1$ have valuations in this range. In this case, 
write $q_{k}(\underline{\theta})=\frac{1-\underline{\theta}}{1-\underline{\theta}+c}$ and rewrite the condition as:

$$
\frac{E\left[k^{2}\right]-z_{1}}{z_{1}}>1+\frac{c}{1-\underline{\theta}}
$$

For any $c<\underline{\theta}<1$ we can find a distribution such that this condition is satisfied. For instance, suppose we assume that everyone has the same number of friendships $k^{*}$ conditional on not having $k=1$. The distribution of friendships is therefore $p_{1}=\underline{\theta}-c, p_{k^{*}}=1-\underline{\theta}+c p_{k}=0$ for $k \neq 1, k^{*}$. Then $\frac{E\left[k^{2}\right]-z_{1}}{z_{1}}=$ $\frac{(1-\underline{\theta}+c) k^{*}\left(k^{*}-1\right)}{\underline{\theta}-c+(1-\underline{\theta}+c) k^{*}}$ and

$$
\lim _{k^{*} \rightarrow \infty} \frac{(1-\underline{\theta}+c) k^{*}\left(k^{*}-1\right)}{\underline{\theta}-c+(1-\underline{\theta}+c) k^{*}}=\infty .
$$

First, I show that demand will be linear in the region $P \in[c, \underline{\theta}]$. Consider

$$
\begin{aligned}
D & =1-H_{0}(1, P) \\
\frac{d D}{d P} & =-\frac{d H_{0}(1, P)}{d P}=-\frac{d\left(1-\sum p_{k} q_{k}(P)\left(1-u(P)^{k}\right)\right)}{d P} \\
& =-(1-u)+\frac{d u}{d P} \sum k p_{k} q_{k}(P) u^{k-1}
\end{aligned}
$$

In the range of prices $P \in[c, \underline{\theta}], \frac{d q_{k}}{d P}=0$ for $k \neq 1$ and $\frac{d q_{1}}{d P}=-\frac{1}{p_{1}}$ for $k=1$ because all consumers $\theta \in[c, \underline{\theta}]$ have $k=1$. Now consider the self consistency relationship for $u(P)$ :

$$
u=1-\frac{1}{z_{1}} \sum_{k=2}^{\infty} k p_{k} q_{k}\left(1-u^{k-1}\right)
$$

This is independent of $q_{1}$. Thus, $\frac{d u}{d P}=0$ for $P \in[c, \underline{\theta}], \frac{d D}{d P}=-(1-u)$, and $D(P)$ is linear. Denote $\underline{u}=u(P)$ for $P \in[c, \underline{\theta}]$. Consider the first-order condition of the monopolist for $P \in[c, \underline{\theta}]$ :

$$
\frac{d \pi}{d P}=D(P)-(P-c)(1-\underline{u})
$$

This is decreasing in $P$ and positive if $\frac{D(P)}{1-\underline{u}}>P-c$. Therefore, the optimal price cannot be less than or equal to $\frac{1+c}{2}$ if $\frac{D\left(\frac{1+c}{2}\right)}{1-\underline{u}}>\frac{1-c}{2}$. We can use the linearity of $D(P)$ for $P \in[c, \underline{\theta}]$ to write $D\left(\frac{1+c}{2}\right)=$ $D(\underline{\theta})-(1-\underline{u})\left(\underline{\theta}-\frac{1-\bar{c}}{2}\right)$ and substitute this in to get the following condition:

$$
\underline{\theta}+\frac{D(\underline{\theta})}{1-\underline{u}}>1
$$

When $P^{\text {crit }}>\underline{\theta} \Leftrightarrow \underline{u}<1$, this can be rewritten using $\sum p_{k} q_{k}=1-\underline{\theta}$

$$
\begin{aligned}
\sum p_{k} q_{k}\left(1-\underline{u}^{k}\right)-(1-\underline{\theta})(1-\underline{u}) & >0 \\
\sum p_{k} q_{k}\left(\underline{u}-\underline{u}^{k}\right) & >0
\end{aligned}
$$

This is true for $\underline{u}<1$. Hence, the monopoly price is greater than $\frac{1+c}{2}$. 


\section{Proof of Theorem 5}

Proof. In the case of perfect assortativity $r=1$ there are two separate populations. In this extreme case no type is friends with an individual of a different type. As a result, demand can be characterized as the sum of the demand curves in each population. These may be calculated separately because neither population is connected to the other. In particular, it is immediate that $D(P, 1)>0, \frac{d D(P, 1)}{d P}=0$ for $P \in\left[\frac{E[k]}{E\left[k^{2}\right]-E[k]} \tilde{\theta}, \tilde{\theta}\right]$. It suffices to show that $\lim _{r \rightarrow 1^{-}} D(P, r)=D(P, 1)$. The primitives are

$$
\begin{aligned}
{\left[\begin{array}{l}
G_{0}^{1}\left(x_{1}, x_{2}\right) \\
G_{0}^{2}\left(x_{1}, x_{2}\right)
\end{array}\right]=\left[\begin{array}{c}
\sum_{k} p_{k}\left(\frac{e_{11} x_{1}+e_{12} x_{2}}{e_{11}+e_{12}}\right)^{k} \\
\sum_{k} p_{k}\left(\frac{e_{21} x_{1}+e_{22} x_{2}}{e_{21}+e_{22}}\right)^{k}
\end{array}\right] } \\
{\left[\begin{array}{l}
G_{0}^{1}\left(x_{1}, x_{2}\right) \\
G_{0}^{2}\left(x_{1}, x_{2}\right)
\end{array}\right]=\left[\begin{array}{c}
\sum_{k} p_{k}\left(\frac{(\tilde{\theta}-e) x_{1}+e x_{2}}{\tilde{\theta}}\right)^{k} \\
\sum_{k} p_{k}\left(\frac{e x_{1}+(1-\tilde{\theta}-e) x_{2}}{1-\tilde{\theta}}\right)^{k}
\end{array}\right] } \\
{\left[\begin{array}{l}
G_{1}^{1}\left(x_{1}, x_{2}\right) \\
G_{1}^{2}\left(x_{1}, x_{2}\right)
\end{array}\right]=\left[\begin{array}{c}
\frac{1}{z_{1}} \sum_{k} k p_{k}\left(\frac{(\tilde{\theta}-e) x_{1}+e x_{2}}{\tilde{\theta}}\right)^{k-1} \\
\frac{1}{z_{1}} \sum_{k} k p_{k}\left(\frac{e x_{1}+(1-\tilde{\theta}-e) x_{2}}{1-\tilde{\theta}}\right)^{k-1}
\end{array}\right] }
\end{aligned}
$$

Note that it is equivalent to consider $e \rightarrow 0^{+}$rather than $r \rightarrow 1^{-}$. Demand is described by the following two equations

$$
\begin{aligned}
{\left[\begin{array}{l}
u_{1}(P, e) \\
u_{2}(P, e)
\end{array}\right]=} & {\left[\begin{array}{c}
\min \left\{\frac{P}{\tilde{\theta}}, 1\right\}+\left(\max \left\{1-\frac{P}{\tilde{\theta}}, 0\right\}\right) \frac{1}{z_{1}} \sum_{k} k p_{k}\left(\frac{(\tilde{\theta}-e) u_{1}(P, e)+e u_{2}(P, e)}{\tilde{\theta}}\right)^{k-1} \\
\max \left\{\frac{P-\tilde{\theta}}{1-\tilde{\theta}}, 0\right\}+\left(\min \left\{\frac{1-P}{1-\tilde{\theta}}, 1\right\}\right) \frac{1}{z_{1}} \sum_{k} k p_{k}\left(\frac{e u_{1}(P, e)+(1-\tilde{\theta}-e) u_{2}(P, e)}{1-\tilde{\theta}}\right)^{k-1}
\end{array}\right] } \\
D(P)= & \max \{\tilde{\theta}-P, 0\} \sum_{k} p_{k}\left(\frac{(\tilde{\theta}-e) u_{1}(P, e)+e u_{2}(P, e)}{\tilde{\theta}}\right)^{k} \\
& +(\min \{1-P, 1-\tilde{\theta}\}) \sum_{k} p_{k}\left(\frac{e u_{1}(P, e)+(1-\tilde{\theta}-e) u_{2}(P, e)}{1-\tilde{\theta}}\right)^{k}
\end{aligned}
$$

It is immediate that $D$ is continuous in $e, u_{1}$, and $u_{2}$ in equation 32 . It remains to check that $u_{1}(e), u_{2}(e)$ are continuous in $e$. In equation 31

$$
\lim _{e \rightarrow 0}\left[\begin{array}{l}
u_{1}(P, e) \\
u_{2}(P, e)
\end{array}\right]=\left[\begin{array}{l}
u_{1}(P, 0) \\
u_{2}(P, 0)
\end{array}\right]
$$

where

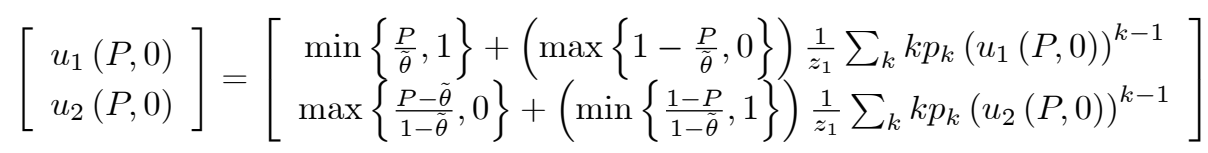


Note, that for $P \in[\tilde{\theta}, 1], u_{1}(0)=u_{1}(e)=1$. In this case, the self-consistency condition for $u_{2}(e)$ is

$$
u_{2}(P, e)=\frac{P-\tilde{\theta}}{1-\tilde{\theta}}+\frac{1-P}{1-\tilde{\theta}} \frac{1}{z_{1}} \sum_{k} k p_{k}\left(\frac{e\left(1-u_{2}(P, e)\right)}{1-\tilde{\theta}}+u_{2}(P, e)\right)^{k-1}
$$

and $u_{2}(e)>u_{2}(0)$. We need to show that for any $u_{2}(0)<w<1, \exists e^{*}>0$ such that for $0<e<e^{*}$

$$
x>\frac{P-\tilde{\theta}}{1-\tilde{\theta}}+\frac{1-P}{1-\tilde{\theta}} \frac{1}{z_{1}} \sum_{k} k p_{k}\left(\frac{e(1-w)}{1-\tilde{\theta}}+w\right)^{k-1}
$$

Now note that $\frac{P-\tilde{\theta}}{1-\tilde{\theta}}+\frac{1-P}{1-\tilde{\theta}} \frac{1}{z_{1}} \sum_{k} k p_{k} x^{k-1}$ is continuous, increasing and convex in $x$, is equal to 1 at $x=1$ and for $u_{2}(0)<1$ the slope is $\frac{1-P}{1-\tilde{\theta}} \frac{1}{z_{1}} \sum_{k} k(k-1) p_{k}\left(u_{2}(0)\right)^{k-2}<1$. Hence, $\frac{P-\tilde{\theta}}{1-\tilde{\theta}}+\frac{1-P}{1-\tilde{\theta}} \frac{1}{z_{1}} \sum_{k} k p_{k}(x)^{k-1}<x$ for all $u_{2}(P, 0)<x<1$, and we can always choose $e$ so that the condition in equation 36 is satisfied.

For $P \in[0, \tilde{\theta}]$

$$
\left[\begin{array}{c}
u_{1}(P, e) \\
u_{2}(P, e)
\end{array}\right]=\frac{\begin{array}{c}
\frac{P}{\tilde{\theta}}+ \\
\left(1-\frac{P}{\tilde{\theta}}\right) \frac{1}{z_{1}} \sum_{k} k p_{k}\left(\frac{(\tilde{\theta}-e) u_{1}(P, e)+e u_{2}(P, e)}{\tilde{\theta}}\right)^{k-1}
\end{array}}{\frac{1}{z_{1}} \sum_{k} k p_{k}\left(\frac{e u_{1}(P, e)+(1-\tilde{\theta}-e) u_{2}(P, e)}{1-\tilde{\theta}}\right)^{k-1}}
$$

where $u_{2}(P, e)<u_{1}(P, e), u_{1}(P, e)<u_{1}(P, 0)$ and $u_{2}(P, e)>u_{2}(P, 0)$. We need to show that for any $x, y$ where $0<y<u_{1}(0), u_{2}(0)<x<1, \exists e^{*}>0$ such that for $0<e<e^{*}$

$$
\begin{aligned}
& y<\frac{P}{\tilde{\theta}}+\left(1-\frac{P}{\tilde{\theta}}\right) \frac{1}{z_{1}} \sum_{k} k p_{k}\left(y+\frac{e(w-y)}{\tilde{\theta}}\right)^{k-1} \\
& x>\frac{1}{z_{1}} \sum_{k} k p_{k}\left(w+\frac{e(y-w)}{1-\tilde{\theta}}\right)^{k-1}
\end{aligned}
$$

where we note that $\frac{1}{z_{1}} \sum_{k} k p_{k} x^{k-1}$ and $\frac{P}{\tilde{\theta}}+\left(1-\frac{P}{\tilde{\theta}}\right) \frac{1}{z_{1}} \sum_{k} k p_{k} x^{k-1}$ are continuous, increasing and convex in $x$ with slopes $<1$ at $u_{2}(P, 0), u_{1}(P, 0)$ respectively. Therefore, we find that

$$
\begin{aligned}
& y<\frac{P}{\tilde{\theta}}+\left(1-\frac{P}{\tilde{\theta}}\right) \frac{1}{z_{1}} \sum_{k} k p_{k} y^{k-1} \text { for all } 0<y<u_{1}(0) \\
& x>\frac{1}{z_{1}} \sum_{k} k p_{k} x^{k-1} \text { for all } u_{2}(0)<x<1
\end{aligned}
$$

Hence, we can find $e^{*}$ such that conditions in equation 38 are satisfied.

\section{Proof of Theorem 6}

Proof. Elasticity is given by

$$
\frac{P}{D(P)} \frac{d D}{d P}=-\frac{P}{1-P}\left[1+\frac{1}{1-\sum_{k, t} p_{k, t} u^{k} v^{2 t}}\left(\frac{d u}{d P} \sum_{k, t} k p_{k, t} u^{k-1} v^{2 t}+\frac{d v}{d P} \sum_{k, t} 2 t p_{k, t} u^{k} v^{2 t-1}\right)\right]
$$


We need to verify $\frac{d u}{d P}, \frac{d v}{d P}>0$ for the proposition to hold. The self consistency condition for $u$ and $v$ is:

$$
\left[\begin{array}{l}
u \\
v
\end{array}\right]=\left[\begin{array}{c}
P+(1-P) \sum_{k, t} \frac{k p_{k, t}}{\sum_{k, t} k p_{k, t}} u^{k-1} v^{2 t} \\
P+(1-P) \sum_{k, t} \frac{t p_{k, t}}{\sum_{k, t} t p_{k, t}} u^{k} v^{2(t-1)}
\end{array}\right]
$$

Now treating each condition separately, denote the values $u_{1}(v, P)$ that satisfy the first equation and the values $v_{2}(u, P)$ which satisfy the second. For $P<P^{c r i t} u_{1}(1, P)<1, v_{2}(1, P)<1, \frac{d u_{1}}{d v}, \frac{d v_{2}}{d u}>0$ and $\frac{d u_{1}}{d v}, \frac{d v_{2}}{d u}$. If there is more than one solution, the solutions can be ordered from highest to lowest. Also, we are guaranteed at least one solution by straightforward application of a fixed point theorem on $[0,1-\varepsilon] \times[0,1-\varepsilon]$ for small $\varepsilon$ when $P<P^{c r i t}$. The highest solution has $\frac{d u_{1}}{d v} \frac{d v_{2}}{d u}<1$ at $\left(u^{*}, v^{*}\right)$. If there exists a second highest solution then $\frac{d u_{1}}{d v} \frac{d v_{2}}{d u} \geq 1$. Note that:

$$
\frac{d u_{1}}{d v} \frac{d v_{2}}{d u}=\frac{(1-P) \sum_{k, t} 2 t \frac{k p_{k, t}}{z_{1 k}} u^{k-1} v^{2 t-1}}{1-(1-P) \sum_{k, t}(k-1) \frac{k p_{k, t}}{z_{1 k}} u^{k-2} v^{2 t}} \frac{(1-P) \sum_{k, t} k \frac{t p_{k, t}}{z_{1 t}} u^{k-1} v^{2(t-1)}}{1-(1-P) \sum_{k, t} 2(t-1) \frac{t p_{k, t}}{z_{1 t}} u^{k} v^{2 t-3}}
$$

is increasing in $u$ and $v$. Hence, for a lower solution we would also have $\frac{d u_{1}}{d v} \frac{d v_{2}}{d u}<1$ which is a contradiction that the lower point is a solution. Hence any solution $\left(u^{*}, v^{*}\right) \in(0,1) \times(0,1)$ is unique. Finally using the implicit function theorem on the equations for $u, v$ we find the result:

$$
\begin{aligned}
& \operatorname{sign}\left(\frac{d v}{d P}\right)=\operatorname{sign}\left(\frac{d u}{d P}\right) \\
& =\operatorname{sign}\left(\frac{-\left|\begin{array}{cc}
-(1-P) \sum_{k, t} k \frac{t p_{k, t}}{z_{1 t}} u^{k-1} v^{2(t-1)} & -\left(1-\sum_{k, t} \frac{k p_{k, t}}{z_{1 k}} u^{k-1} v^{2 t}\right) \\
1-(1-P) \sum_{k, t} 2(t-1) \frac{t p_{k, t}}{z_{1 t}} u^{k} v^{2 t-3} & -\left(1-\sum_{k, t} \frac{k p_{k, t}}{z_{1 k}} u^{k-1} v^{2(t-1)}\right)
\end{array}\right|}{\left|\begin{array}{cc}
-(1-P) \sum_{k, t} k \frac{t p_{k, t}}{z_{1 t}} u^{k-1} v^{2(t-1)} & 1-(1-P) \sum_{k, t}(k-1) \frac{k p_{k, t}}{z_{1 k}} u^{k-2} v^{2 t} \\
1-(1-P) \sum_{k, t} 2(t-1) \frac{t p_{k, t}}{z_{1 t}} u^{k} v^{2 t-3} & -(1-P) \sum_{k, t} 2 t \frac{k p_{k, t}}{z_{1 k}} u^{k-1} v^{2 t-1}
\end{array}\right|}\right)
\end{aligned}
$$

where the numerator is positive and the denominator is

$$
\begin{aligned}
= & {\left[1-(1-P) \sum_{k, t} 2(t-1) \frac{t p_{k, t}}{z_{1 t}} u^{k} v^{2 t-3}\right]\left[1-(1-P) \sum_{k, t}(k-1) \frac{k p_{k, t}}{z_{1 k}} u^{k-2} v^{2 t}\right] } \\
& -\left[(1-P) \sum_{k, t} k \frac{t p_{k, t}}{z_{1 t}} u^{k-1} v^{2(t-1)}\right]\left[(1-P) \sum_{k, t} 2 t \frac{k p_{k, t}}{z_{1 k}} u^{k-1} v^{2 t-1}\right] \\
> & 0
\end{aligned}
$$

the final inequality follows from $\frac{d u_{1}}{d v} \frac{d v_{2}}{d u}<1$ at $\left(u^{*}, v^{*}\right)$

\section{$9 \quad$ Proof of Theorem 7}

Proof. Let $v$ be the probability that an edge of a triad is connected to the giant component then:

$$
\begin{aligned}
v^{2} & =\left(P+(1-P) \sum \frac{\frac{k}{2} p_{k}}{\frac{z_{1}}{2}}\left(v^{2}\right)^{\frac{k}{2}-1}\right)^{2} \\
v & =P+(1-P) \sum \frac{k p_{k}}{z_{1}}(v)^{k-2}
\end{aligned}
$$


We can compare this to the probability $u$ that a regular friendships is connected to the giant component:

$$
u=P+(1-P) \sum \frac{k p_{k}}{z_{1}}(u)^{k-1}
$$

hence $v>u$ and

$$
\begin{aligned}
(1-P)\left(1-\sum_{t} p_{t}\left(v^{2}\right)^{t}\right) & =(1-P)\left(1-\sum_{k} p_{k} v^{k}\right) \\
(1-P)\left(1-\sum_{k} p_{k} v^{k}\right) & <(1-P)\left(1-\sum_{k} p_{k} u^{k}\right) \\
D^{\text {tri }} & <D^{\text {reg }}
\end{aligned}
$$

where the first line comes from $p_{k}=p_{t}$ for $k=2 t$, second line follows from $v>u$ and the third from the definition of demand.

\section{Proof of Theorem 8}

Proof. When the number of friends and valuations are independent we can write demand as:

$$
D(P, \omega)=(1-P)\left(1-(1-\omega) \sum_{k} p_{k}\left(H_{1}(1-\omega, P)\right)^{k}\right)
$$

and the elasticity as:

$$
\begin{aligned}
\frac{P}{D} \frac{d D}{d P}= & \frac{P}{(1-P)\left(1-(1-\omega) \sum_{k} p_{k}\left(H_{1}(1-\omega, P)\right)^{k}\right)} \times \\
& {\left[\begin{array}{c}
-\left(1-(1-\omega) \sum_{k} p_{k}\left(H_{1}(1-\omega, P)\right)^{k}\right) \\
-(1-P)(1-\omega) \frac{d H_{1}(1-\omega, P)}{d P} \sum_{k} k p_{k}\left(H_{1}(1-\omega, P)\right)^{k-1}
\end{array}\right] } \\
= & -\frac{P}{1-P}\left[1+(1-P)(1-\omega) \frac{d H_{1}(1-\omega, P)}{d P} \frac{\sum_{k} k p_{k}\left(H_{1}(1-\omega, P)\right)^{k-1}}{\left(1-(1-\omega) \sum_{k} p_{k}\left(H_{1}(1-\omega, P)\right)^{k}\right)}\right]
\end{aligned}
$$

where the second term inside the brackets is positive. Note:

$$
\frac{d H_{1}(1-\omega, P)}{d P}=\frac{\alpha\left(1-(1-\omega) \sum_{k} \frac{k p_{k}}{z_{1}}\left(H_{1}(1-\omega, P)\right)^{k-1}\right)}{1-\alpha(1-\omega)(1-P) \sum_{k} \frac{k(k-1) p_{k}}{z_{1}}\left(H_{1}(1-\omega, P)\right)^{k-2}}>0
$$


We check that there is a unique solution to the first order condition and it is sufficient:

$$
\begin{aligned}
& \frac{\partial\left(\frac{P}{D} \frac{d D}{d P}\right)}{\partial P}=-\left(\frac{1}{1-P}+\frac{P}{(1-P)^{2}}\right)\left[\begin{array}{c}
1+(1-P)(1-\omega) \frac{\alpha\left(1-(1-\omega) \sum_{k} \frac{k p_{k}}{z_{1}}\left(H_{1}(1-\omega, P)\right)^{k-1}\right)}{1-\alpha(1-\omega)(1-P) \sum_{k} \frac{k(k-1) p_{k}}{z_{1}\left(H_{1}(1-\omega, P)\right)^{k-2}}} \\
\times \frac{\sum_{k} k p_{k}\left(H_{1}(1-\omega, P)\right)^{k-1}}{\left(1-(1-\omega) \sum_{k} p_{k}\left(H_{1}(1-\omega, P)\right)^{k}\right)}
\end{array}\right] \\
& +\frac{P}{1-P}\left[\begin{array}{c}
(1-\omega) \frac{\alpha\left(1-(1-\omega) \sum_{k} \frac{k p_{k}}{z_{1}}\left(H_{1}(1-\omega, P)\right)^{k-1}\right)}{1-\alpha(1-\omega)(1-P) \sum_{k} \frac{k(k-1) p_{k}}{z_{1}}\left(H_{1}(1-\omega, P)\right)^{k-2}} \\
\times \frac{\sum_{k} k p_{k}\left(H_{1}(1-\omega, P)\right)^{k-1}}{\left(1-(1-\omega) \sum_{k} p_{k}\left(H_{1}(1-\omega, P)\right)^{k}\right)}
\end{array}\right] \\
& -P(1-\omega) \alpha \frac{d H_{1}}{d P} \times \\
& \begin{array}{c}
{\left[\begin{array}{c}
\frac{-(1-\omega) \sum_{k} \frac{k p_{k}}{z_{1}}(k-1)\left(H_{1}(1-\omega, P)\right)^{k-2}}{1-\alpha(1-\omega)(1-P) \sum_{k} \frac{k(k-1) p_{k}}{z_{1}}\left(H_{1}(1-\omega, P)\right)^{k-2}} \frac{\sum_{k} k p_{k}\left(H_{1}(1-\omega, P)\right)^{k-1}}{\left(1-(1-\omega) \sum_{k} p_{k}\left(H_{1}(1-\omega, P)\right)^{k}\right)} \\
+\frac{\left(1-(1-\omega) \sum_{k} \frac{k p_{k}}{z_{1}}\left(H_{1}(1-\omega, P)\right)^{k-1}\right)}{1-\alpha(1-\omega)(1-P) \sum_{k} \frac{k(k-1) p_{k}}{z_{1}}\left(H_{1}(1-\omega, P)\right)^{k-2}} \frac{\sum_{k} k p_{k}(k-1)\left(H_{1}(1-\omega, P)\right)^{k-2}}{\left(1-(1-\omega) \sum_{k} p_{k}\left(H_{1}(1-\omega, P)\right)^{k}\right)} \\
+\alpha(1-\omega)(1-P) \sum_{k} \frac{k(k-1)(k-2) p_{k}}{z_{1}}\left(H_{1}(1-\omega, P)\right)^{k-3} \\
\times \frac{\left(1-(1-\omega) \sum_{k} \frac{k p_{k}}{z_{1}}\left(H_{1}(1-\omega, P)\right)^{k-1}\right)}{\left(1-\alpha(1-\omega)(1-P) \sum_{k} \frac{k(k-1) p_{k}}{z_{1}}\left(H_{1}(1-\omega, P)\right)^{k-2}\right)^{2}} \frac{\sum_{k} k p_{k}\left(H_{1}(1-\omega, P)\right)^{k-1}}{\left(1-(1-\omega) \sum_{k} p_{k}\left(H_{1}(1-\omega, P)\right)^{k}\right)} \\
+(1-\omega) \sum_{k} k p_{k}\left(H_{1}(1-\omega, P)\right)^{k-1} \\
\times \frac{\alpha\left(1-(1-\omega) \sum_{k} \frac{k p_{k}}{z_{1}}\left(H_{1}(1-\omega, P)\right)^{k-1}\right)}{\left.x_{k}\right)} \frac{\sum_{k} k p_{k}\left(H_{1}(1-\omega, P)\right)^{k-1}}{\left(1-\alpha(1-\omega)(1-P) \sum_{k} \frac{k(k-1) p_{k}}{z_{1}}\left(H_{1}(1-\omega, P)\right)^{k-2} p_{k}\left(H_{1}(1-\omega, P)\right)^{k}\right)^{2}}
\end{array}\right]}
\end{array}
\end{aligned}
$$

The first two lines are negative. For $\alpha$ small enough the entire term is negative because $\frac{d H_{1}}{d P}$ is linear in $\alpha$ thus the term $-P(1-\omega) \alpha \frac{d H_{1}}{d P}$ is $o\left(\alpha^{2}\right)$. The first two lines are $o(\alpha)$ hence these terms determine the sign of the whole expression in the limit of small $\alpha$. Hence there is a single solution to the first order condition for the price. A similar condition may be derived for the case of clustering.

i) We want to show that for two networks $\left\{p_{k}^{\prime}\right\}$ and $\left\{p_{k}^{\prime \prime}\right\}$ where $z_{1}^{\prime}>z_{1}^{\prime \prime}$ that

$$
\begin{gathered}
\lim _{\alpha \rightarrow 0} \frac{\alpha\left(1-(1-\omega) \sum_{k} \frac{k p_{k}^{\prime}}{z_{1}^{\prime}}\left(H_{1}^{\prime}(1-\omega, P)\right)^{k-1}\right)}{1-\alpha(1-\omega)(1-P) \sum_{k} \frac{k(k-1) p_{k}^{\prime}}{z_{1}^{\prime}}\left(H_{1}^{\prime}(1-\omega, P)\right)^{k-2}} \frac{\sum_{k} k p_{k}^{\prime}\left(H_{1}^{\prime}(1-\omega, P)\right)^{k-1}}{\left(1-(1-\omega) \sum_{k} p_{k}^{\prime}\left(H_{1}^{\prime}(1-\omega, P)\right)^{k}\right)}(49) \\
>\lim _{\alpha \rightarrow 0} \frac{\alpha\left(1-(1-\omega) \sum_{k} \frac{k p_{k}^{\prime \prime}}{z_{1}^{\prime \prime}}\left(H_{1}^{\prime \prime}(1-\omega, P)\right)^{k-1}\right)}{1-\alpha(1-\omega)(1-P) \sum_{k} \frac{k(k-1) p_{k}^{\prime \prime}}{z_{1}^{\prime \prime}}\left(H_{1}^{\prime \prime}(1-\omega, P)\right)^{k-2}} \frac{\sum_{k} k p_{k}^{\prime \prime}\left(H_{1}^{\prime \prime}(1-\omega, P)\right)^{k-1}}{\left(1-(1-\omega) \sum_{k} p_{k}^{\prime \prime}\left(H_{1}^{\prime \prime}(1-\omega, P)\right)^{k}\right)}
\end{gathered}
$$

now $\lim _{\alpha \rightarrow 0} H_{1}^{\prime}(1-\omega, P)=\lim _{\alpha \rightarrow 0} H_{1}^{\prime \prime}(1-\omega, P)=1$ and noting that

$$
\lim _{\alpha \rightarrow 0} \frac{\left(1-(1-\omega) \sum_{k} \frac{k p_{k}}{z_{1}}\left(H_{1}(1-\omega, P)\right)^{k-1}\right)}{1-\alpha(1-\omega)(1-P) \sum_{k} \frac{k(k-1) p_{k}}{z_{1}}\left(H_{1}(1-\omega, P)\right)^{k-2}} \frac{1}{\left(1-(1-\omega) \sum_{k} p_{k}\left(H_{1}(1-\omega, P)\right)^{k}\right)}=1
$$

then we can choose $\alpha$ so that the term above is arbitrarily close to 1 . When this is the case the more elastic demand is determined by the term $\lim _{\alpha \rightarrow 0} \sum_{k} k p_{k}\left(H_{1}(1-\omega, P)\right)^{k-1}=\sum_{k} k p_{k}$ which is greater by assumption for $\left\{p_{k}^{\prime}\right\}$. 
ii) To find the effect of mean-preserving spreads we must consider the effects of second order terms. We can take a Taylor series approximation at $\alpha=0$ to find the relative size of the second order term for small values of $\alpha$. Letting

$$
f(\alpha)=\frac{\alpha\left(1-(1-\omega) \sum_{k} \frac{k p_{k}}{z_{1}}(u(\alpha))^{k-1}\right)}{1-\alpha(1-\omega)(1-P) \sum_{k} \frac{k(k-1) p_{k}}{z_{1}}(u(\alpha))^{k-2}} \frac{\sum_{k} k p_{k}(u(\alpha))^{k-1}}{\left(1-(1-\omega) \sum_{k} p_{k}(u(\alpha))^{k}\right)}
$$

Now

$$
\frac{d f}{d \alpha}=\frac{\partial f}{\partial \alpha}+\frac{\partial f}{\partial u} \frac{d u}{d \alpha}
$$

Direct effect $\frac{\partial f}{\partial \alpha}$ :

$$
\begin{aligned}
& \frac{\partial f}{\partial \alpha}= \frac{\left(1-(1-\omega) \sum_{k} \frac{k p_{k}}{z_{1}}(u)^{k-1}\right)}{1-\alpha(1-\omega)(1-P) \sum_{k} \frac{k(k-1) p_{k}}{z_{1}}(u)^{k-2}} \frac{\sum_{k} k p_{k}(u)^{k-1}}{\left(1-(1-\omega) \sum_{k} p_{k}(u)^{k}\right)} \\
&+(1-\omega)(1-P) \sum_{k} \frac{k(k-1) p_{k}}{z_{1}}(u)^{k-2} \\
& \times \frac{\alpha\left(1-(1-\omega) \sum_{k} \frac{k p_{k}}{z_{1}}(u)^{k-1}\right)}{\left(1-\alpha(1-\omega)(1-P) \sum_{k} \frac{k(k-1) p_{k}}{z_{1}}(u)^{k-2}\right)^{2}} \frac{\sum_{k} k p_{k}(u)^{k-1}}{\left(1-(1-\omega) \sum_{k} p_{k}(u)^{k}\right)} \\
&\left.\frac{\partial f}{\partial \alpha}\right|_{\alpha=0}=z_{1}
\end{aligned}
$$

Indirect effect $\frac{\partial f}{\partial u} \frac{d u}{d \alpha}$

$$
\frac{\partial f}{\partial u} \frac{d u}{d \alpha}=\alpha \frac{d u}{d \alpha}\left[\begin{array}{c}
\frac{-(1-\omega) \sum_{k} \frac{k(k-1) p_{k}}{z_{1}}(u)^{k-2}}{1-\alpha(1-\omega)(1-P) \sum_{k} \frac{k(k-1) p_{k}}{z_{1}}(u)^{k-2}} \frac{\sum_{k} k p_{k}(u)^{k-1}}{\left(1-(1-\omega) \sum_{k} p_{k}(u)^{k}\right)} \\
+\frac{\left(1-(1-\omega) \sum_{k} \frac{k p_{k}}{z_{1}}(u)^{k-1}\right)}{1-\alpha(1-\omega)(1-P) \sum_{k} \frac{k(k-1) p_{k}}{z_{1}}(u)^{k-2}} \frac{\sum_{k} k(k-1) p_{k}(u)^{k-2}}{\left(1-(1-\omega) \sum_{k} p_{k}(u)^{k}\right)} \\
\times \frac{\left(1-(1-\omega) \sum_{k} \frac{k p_{k}}{z_{1}}(u)^{k-1}\right)}{\underbrace{}_{1} \frac{k(k-1)(k-2) p_{k}}{z_{1}}(u)^{k-3}} \frac{\sum_{k} k p_{k}(u)^{k-1}}{\left(1-\alpha(1-\omega)(1-P) \sum_{k} \frac{k(k-1) p_{k}}{z_{1}}(u)^{k-2}\right)^{2}} \frac{\left(1-(1-\omega) \sum_{k} p_{k}(u)^{k}\right)}{(1-\omega) \sum_{k} k p_{k}(u)^{k-1}} \\
\times \frac{\left(1-(1-\omega) \sum_{k} \frac{k p_{k}}{\left.z_{1}(u)^{k-1}\right)}\right.}{\left(1-\alpha(1-\omega)(1-P) \sum_{k} \frac{k(k-1) p_{k}}{z_{1}}(u)^{k-2}\right)} \frac{\sum_{k} k p_{k}(u)^{k-1}}{\left(1-(1-\omega) \sum_{k} p_{k}(u)^{k}\right)^{2}}
\end{array}\right]
$$

where

$$
u=1-\alpha(1-P)+\alpha(1-\omega)(1-P) \sum \frac{k p_{k}}{z_{1}} u^{k-1}
$$

hence

$$
\begin{aligned}
\frac{d u}{d \alpha} & =-\frac{(1-P)\left(1-(1-\omega) \sum \frac{k p_{k}}{z_{1}} u^{k-1}\right)}{1-\alpha(1-\omega)(1-P) \sum \frac{k(k-1) p_{k}}{z_{1}} u^{k-2}} \\
\left.\frac{d u}{d \alpha}\right|_{\alpha=0} & =-\omega(1-P)
\end{aligned}
$$


and evaluating

$$
\frac{\partial f}{\partial u} \frac{d u}{d \alpha}{ }_{\alpha=0}=0
$$

therefore

$$
\frac{d f}{d \alpha}_{\alpha=0}=z_{1}
$$

To consider a mean-preserving spread we must check the second order terms $\frac{d^{2} f}{d \alpha^{2}}$. We can ignore any terms that are linear in $\alpha$ as these terms are otherwise finite and are thus zero in the limit $\alpha \rightarrow 0$. Ignoring these terms, the remaining terms are:

$$
\begin{aligned}
& \frac{d^{2} f}{d \alpha^{2}}=2 \frac{d u}{d \alpha}\left[\begin{array}{c}
\frac{-(1-\omega) \sum_{k} \frac{k(k-1) p_{k}}{z_{1}}(u)^{k-2}}{1-\alpha(1-\omega)(1-P) \sum_{k} \frac{k(k-1) p_{k}}{z_{1}}(u)^{k-2}} \frac{\sum_{k} k p_{k}(u)^{k-1}}{\left(1-(1-\omega) \sum_{k} p_{k}(u)^{k}\right)} \\
+\frac{\left(1-(1-\omega) \sum_{k} \frac{k p_{k}}{z_{1}}(u)^{k-1}\right)}{1-\alpha(1-\omega)(1-P) \sum_{k} \frac{k(k-1) p_{k}(u)^{k-2}}{z_{k}} \frac{\sum_{k} k(k-1) p_{k}(u)^{k-2}}{\left(1-(1-\omega) \sum_{k} p_{k}(u)^{k}\right)}} \\
\times \frac{\left(1-(1-\omega) \sum_{k} \frac{k p_{k}}{z_{1}}(u)^{k-1}\right)}{\underbrace{}_{1} \frac{k(k-1)(k-2) p_{k}}{z_{1}}(u)^{k-3}} \frac{\sum_{k} k p_{k}(u)^{k-1}}{\left(1-\alpha(1-\omega)(1-P) \sum_{k} \frac{k(k-1) p_{k}}{z_{1}}(u)^{k-2}\right)^{2}} \\
+\left(1-(1-\omega) \sum_{k} p_{k}(u)^{k}\right) \\
\times \frac{\left(1-(1-\omega) \sum_{k} \frac{k p_{k}}{z_{1}}(u)_{k}^{k-1}\right)}{\left(1-\alpha(1-\omega)(1-P) \sum_{k} \frac{k(k-1) p_{k}}{z_{1}}(u)^{k-2}\right)} \frac{\sum_{k} k p_{k}(u)^{k-1}}{\left(1-(1-\omega) \sum_{k} p_{k}(u)^{k}\right)^{2}}
\end{array}\right] \\
& +(1-\omega)(1-P) \sum_{k} \frac{k(k-1) p_{k}}{z_{1}}(u)^{k-2} \\
& \times \frac{\left(1-(1-\omega) \sum_{k} \frac{k p_{k}}{z_{1}}(u)^{k-1}\right)}{\left(1-\alpha(1-\omega)(1-P) \sum_{k} \frac{k(k-1) p_{k}}{z_{1}}(u)^{k-2}\right)^{2}} \frac{\sum_{k} k p_{k}(u)^{k-1}}{\left(1-(1-\omega) \sum_{k} p_{k}(u)^{k}\right)}
\end{aligned}
$$

evaluated at $\alpha=0$ and rearranging we find

$$
\begin{aligned}
\left.\frac{d^{2} f}{d \alpha^{2}}\right|_{\alpha=0}= & -2 \omega(1-P)\left[\begin{array}{c}
\frac{-(1-\omega)\left(E\left[k^{2}\right]-z_{1}\right)}{z_{1}} \frac{z_{1}}{\omega} \\
\frac{\omega}{1} \frac{E\left[k^{2}\right]-z_{1}}{\omega} \\
0 \\
(1-\omega) z_{1}\left(\frac{\omega}{1} z_{1}\right.
\end{array}\right] \\
& +\frac{(1-\omega)(1-P)\left(E\left[k^{2}\right]-z_{1}\right)}{z_{1}}\left[\frac{\omega}{1} \frac{z_{1}}{\omega}\right] \\
= & (1-P)\left[\begin{array}{c}
\left.2(1-\omega)\left(E\left[k^{2}\right]-z_{1}\right)-2 \omega\left(E\left[k^{2}\right]-z_{1}\right)-2(1-\omega)\left(z_{1}\right)^{2}\right] \\
+(1-\omega)\left(E\left[k^{2}\right]-z_{1}\right)
\end{array}\right] \\
\left.\frac{d^{2} f}{d \alpha^{2}}\right|_{\alpha=0}= & (1-P)\left[\left(E\left[k^{2}\right]-z_{1}\right)(3-5 \omega)-2(1-\omega)\left(z_{1}\right)^{2}\right]
\end{aligned}
$$

which is increasing in $E\left[k^{2}\right]$ when $\omega<0.6$, hence a mean-preserving spread will increase the elasticity and reduce prices. 
iii) The equations under clustering

$$
\begin{aligned}
v & =1-\alpha(1-P)+\alpha(1-\omega)(1-P) \sum_{k} \frac{k p_{k}}{z_{1}}(v)^{k-2} \\
D(P) & =(1-P)\left(1-(1-\omega) \sum_{k} p_{k} v^{k}\right) \\
\frac{P}{D} \frac{d D}{d P} & =-\frac{P}{1-P}\left(1+(1-P)(1-\omega) \frac{d v}{d P} \frac{\sum_{k} k p_{k} v^{k-1}}{\left(1-(1-\omega) \sum_{k} p_{k} v^{k}\right)}\right)
\end{aligned}
$$

where

$$
\frac{d v}{d P}=\frac{\alpha\left(1-(1-\omega) \sum_{k} \frac{k p_{k}}{z_{1}}(v)^{k-2}\right)}{1-\alpha(1-\omega)(1-P) \sum_{k} \frac{k(k-2) p_{k}}{z_{1}}(v)^{k-3}}
$$

Just considering the final term inside the brackets we define:

$$
f(\alpha)=\frac{\alpha\left(1-(1-\omega) \sum_{k} \frac{k p_{k}}{z_{1}}(v)^{k-2}\right)}{1-\alpha(1-\omega)(1-P) \sum_{k} \frac{k(k-2) p_{k}}{z_{1}}(v)^{k-3}} \frac{\sum_{k} k p_{k} v^{k-1}}{\left(1-(1-\omega) \sum_{k} p_{k} v^{k}\right)}
$$

Direct effect $\frac{\partial f}{\partial \alpha}$ :

$$
\begin{aligned}
\frac{\partial f}{\partial \alpha}= & \frac{\left(1-(1-\omega) \sum_{k} \frac{k p_{k}}{z_{1}}(v)^{k-2}\right)}{1-\alpha(1-\omega)(1-P) \sum_{k} \frac{k(k-2) p_{k}}{z_{1}}(v)^{k-3}} \frac{\sum_{k} k p_{k} v^{k-1}}{\left(1-(1-\omega) \sum_{k} p_{k} v^{k}\right)} \\
& +(1-\omega)(1-P) \sum_{k} \frac{k(k-2) p_{k}}{z_{1}}(v)^{k-3} \\
& \times \frac{\alpha\left(1-(1-\omega) \sum_{k} \frac{k p_{k}}{z_{1}}(v)^{k-2}\right)}{\left(1-\alpha(1-\omega)(1-P) \sum_{k} \frac{k(k-2) p_{k}}{z_{1}}(v)^{k-3}\right)^{2}} \frac{\sum_{k} k p_{k} v^{k-1}}{\left(1-(1-\omega) \sum_{k} p_{k} v^{k}\right)}
\end{aligned}
$$

Indirect effect $\frac{\partial f}{\partial v} \frac{d v}{d \alpha}$ :

$$
\frac{\partial f}{\partial v} \frac{d v}{d \alpha}=\alpha \frac{d v}{d \alpha}\left[\begin{array}{c}
-\frac{(1-\omega) \sum_{k} \frac{k(k-2) p_{k}}{z_{1}} v^{k-3}}{1-\alpha(1-\omega)(1-P) \sum_{k} \frac{k(k-2) p_{k}}{z_{1}} v^{k-3}} \frac{\sum_{k} k p_{k} v^{k-1}}{\left(1-(1-\omega) \sum_{k} p_{k} v^{k}\right)} \\
+\frac{\left(1-(1-\omega) \sum_{k} \frac{k p_{k}}{z_{1}}(v)^{k-2}\right)}{1-\alpha(1-\omega)(1-P) \sum_{k} \frac{k(k-2) p_{k}}{z_{1}}(v)^{k-3}} \frac{\sum_{k} k(k-1) p_{k} v^{k-2}}{\left(1-(1-\omega) \sum_{k} p_{k} v^{k}\right)} \\
+(1-\omega) \sum_{k} p_{k} k v^{k-1} \\
\times \frac{\left(1-(1-\omega) \sum_{k} \frac{k p_{k}}{z_{1}}(v)^{k-2}\right)}{1-\alpha(1-\omega)(1-P) \sum_{k} \frac{k(k-2) p_{k}}{z_{1}}(v)^{k-3} \frac{\sum_{k} k p_{k} v^{k-1}}{\left(1-(1-\omega) \sum_{k} p_{k} v^{k}\right)^{2}}} \\
+\alpha(1-\omega)(1-P) \sum_{k} \frac{k(k-2)(k-3) p_{k}}{z_{1}} v^{k-4} \\
\times \frac{\left(1-(1-\omega) \sum_{k} \frac{k p_{k}}{z_{1}}(v)^{k-2}\right)}{\left(1-\alpha(1-\omega)(1-P) \sum_{k} \frac{k(k-2) p_{k}}{z_{1}} v^{k-3}\right)^{2}} \frac{\sum_{k} k p_{k} v^{k-1}}{\left(1-(1-\omega) \sum_{k} p_{k} v^{k}\right)}
\end{array}\right]
$$

where

$$
\frac{d v}{d \alpha}=-(1-P) \frac{1-(1-\omega) \sum_{k} \frac{k p_{k}}{z_{1}}(v)^{k-2}}{1-\alpha(1-\omega)(1-P) \sum_{k} \frac{k(k-2) p_{k}}{z_{1}}(v)^{k-3}}
$$


first order term

$$
\begin{aligned}
\left.\frac{\partial f}{\partial \alpha}\right|_{\alpha=0} & =\sum_{k} k p_{k} \\
\left.\frac{\partial f}{\partial v} \frac{d v}{d P}\right|_{\alpha=0} & =0
\end{aligned}
$$

is the same with or without clustering. Now considering the second order terms ignoring any that are linear in $\alpha$

$$
\begin{aligned}
& 2 \frac{d v}{d \alpha}\left[\begin{array}{c}
-\frac{(1-\omega) \sum_{k} \frac{k(k-2) p_{k}}{z_{1}} v^{k-3}}{1-\alpha(1-\omega)(1-P) \sum_{k} \frac{k(k-2) p_{k}}{z^{2}} v^{k-3}} \frac{\sum_{k} k p_{k} v^{k-1}}{\left(1-(1-\omega) \sum_{k} p_{k} v^{k}\right)} \\
+\frac{\left(1-(1-\omega) \sum_{k} \frac{k p_{k}}{\left.z_{1}(v)^{k-2}\right)}\right.}{1-\alpha(1-\omega)(1-P) \sum_{k} \frac{k(k-2) p_{k}}{z_{1}}(v)^{k-3}} \frac{\sum_{k} k(k-1) p_{k} v^{k-2}}{\left(1-(1-\omega) \sum_{k} p_{k} v^{k}\right)} \\
+(1-\omega) \sum_{k} p_{k} k v^{k-1} \\
\times \frac{\left(1-(1-\omega) \sum_{k} \frac{k p_{k}}{z_{1}}(v)^{k-2}\right)}{1-\alpha(1-\omega)(1-P) \sum_{k} \frac{k(k-2) p_{k}}{z_{1}}(v)^{k-3}} \frac{\sum_{k} k p_{k} v^{k-1}}{\left(1-(1-\omega) \sum_{k} p_{k} v^{k}\right)^{2}} \\
+\alpha(1-\omega)(1-P) \sum_{k} \frac{k(k-2)(k-3) p_{k}}{z_{1}} v^{k-4} \\
\times \frac{\left(1-(1-\omega) \sum_{k} \frac{k p_{k}}{z_{1}}(v)^{k-2}\right)}{\left(1-\alpha(1-\omega)(1-P) \sum_{k} \frac{k(k-2) p_{k}}{z_{1}} v^{k-3}\right)^{2}} \frac{\sum_{k} k p_{k} v^{k-1}}{\left(1-(1-\omega) \sum_{k} p_{k} v^{k}\right)}
\end{array}\right] \\
& +(1-\omega)(1-P) \sum_{k} \frac{k(k-2) p_{k}}{z_{1}}(v)^{k-3} \\
& \times \frac{\left(1-(1-\omega) \sum_{k} \frac{k p_{k}}{z_{1}}(v)^{k-2}\right)}{\left(1-\alpha(1-\omega)(1-P) \sum_{k} \frac{k(k-2) p_{k}}{z_{1}}(v)^{k-3}\right)^{2}} \frac{\sum_{k} k p_{k} v^{k-1}}{\left(1-(1-\omega) \sum_{k} p_{k} v^{k}\right)} \\
& \left.\frac{d^{2} f}{d \alpha^{2}}\right|_{\alpha=0}=-2(1-P) \omega\left[\begin{array}{c}
-(1-\omega)\left(E\left[k^{2}\right]-2 z_{1}\right) \frac{1}{\omega} \\
+\left(E\left[k^{2}\right]-z_{1}\right) \\
+(1-\omega) z_{1} \frac{1}{\omega} \\
+0
\end{array}\right] \\
& +(1-\omega)(1-P)\left(E\left[k^{2}\right]-2 z_{1}\right) \\
& \left.\frac{d^{2} f}{d \alpha^{2}}\right|_{\alpha=0}=(1-P)\left[\left(E\left[k^{2}\right]-z_{1}\right)(3-5 \omega)-2 \omega(1-\omega) z_{1}\right]-3(1-\omega)(1-P) z_{1}
\end{aligned}
$$

the final term $-3(1-\omega)(1-P) z_{1}<0$. The expression is otherwise identical to the case without clustering, hence clustering results in a higher price.

\section{Proof of Theorem 9}

Proof. Assuming a constant cost per unit of advertising $\alpha$ and marginal cost of production $c$, the monopolist's profit is defined by

$$
\pi(P, \omega)=(P-c)\left(1-H_{0}(1-\omega, P)\right)-\alpha \omega
$$


In a Poisson network the generating functions are $G_{0}(x)=G_{1}(x)=\exp \left(z_{1}(x-1)\right)$; hence the firm's profit is given by

$$
\begin{aligned}
\pi\left(P, z_{1}\right) & =(P-c) D(P, \omega)-\alpha \omega \\
& =(1-P)\left(1-(1-\omega) \exp \left(-z_{1}\left(1-H_{1}(1-\omega, P)\right)\right)\right)
\end{aligned}
$$

and the optimal price and level of advertising $P^{*}, \omega^{*}$ satisfy:

$$
\begin{aligned}
\left.\frac{d \pi(P, \omega)}{d P}\right|_{P=P^{*}, \omega=\omega^{*}} & =0 \\
\left.\frac{d \pi(P, \omega)}{d \omega}\right|_{P=P^{*}, \omega=\omega^{*}} & =0 \\
D\left(P^{*}, \omega^{*}\right)+\left.\left(P^{*}-c\right) \frac{d D}{d P}\right|_{P=P^{*}, \omega=\omega^{*}} & =0 \\
\left.\frac{D\left(P^{*}, \omega^{*}\right)}{P^{*}} \frac{d D}{d P}\right|_{P=P^{*}, \omega=\omega^{*}} & =\frac{\left(P^{*}-c\right)}{P^{*}} \\
\left.\left(P^{*}-c\right) \frac{d D}{d \omega}\right|_{P=P^{*}, \omega=\omega^{*}}-\alpha & =0
\end{aligned}
$$

We need to show

$$
\left.\frac{\partial\left(\left.\frac{D\left(P^{*}, \omega^{*}\right)}{P^{*}} \frac{d D}{d P}\right|_{P=P^{*}, \omega=\omega^{*}}\right)}{\partial \omega}\right|_{P=P^{*}, \omega=\omega^{*}}>0
$$

since $D=1-H_{1}(1-\omega, P)$, we know

$$
\begin{aligned}
H_{1}(1-\omega, P) & =P+(1-P)(1-\omega) \exp \left(-z_{1}\left(1-H_{1}(1-\omega, P)\right)\right) \\
\frac{d H_{1}(1-\omega, P)}{d P} & =\frac{1-(1-\omega) \exp \left(-z_{1}\left(1-H_{1}(1-\omega, P)\right)\right)}{\left(1-(1-\omega)(1-P) z_{1} \exp \left(-z_{1}\left(1-H_{1}(1-\omega, P)\right)\right)\right)}
\end{aligned}
$$

$\frac{d H_{1}}{d P}=-\frac{d D}{d P}$ hence the elasticity of demand with respect to price is

$$
\frac{D(P, \omega)}{P} \frac{d D(P, \omega)}{d P}=-\frac{P}{1-P} \frac{1}{\left(1-(1-\omega)(1-P) z_{1} \exp \left(-z_{1}\left(1-H_{1}(1-\omega, P)\right)\right)\right)}
$$

Holding the price constant, the sign of the derivative w.r.t. $\omega$ is $\operatorname{sign}\left(-\frac{d\left((1-\omega)(1-P) z_{1} \exp \left(-z_{1}\left(1-H_{1}(1-\omega, P)\right)\right)\right)}{d \omega}\right)$

$$
\begin{aligned}
& \frac{d\left((1-\omega)(1-P) z_{1} \exp \left(-z_{1}\left(1-H_{1}(1-\omega, P)\right)\right)\right.}{d \omega} \\
= & -(1-P) z_{1} \exp \left(-z_{1}\left(1-H_{1}(1-\omega, P)\right)\right) \\
& +\frac{d H_{1}}{d \omega}\left((1-\omega)(1-P) z_{1} \exp \left(-z_{1}\left(1-H_{1}(1-\omega, P)\right)\right)\right)
\end{aligned}
$$

Hence

$$
\left.\frac{\partial\left(\left.\frac{D\left(P^{*}, \omega^{*}\right)}{P^{*}} \frac{d D}{d P}\right|_{P=P^{*}, \omega=\omega^{*}}\right)}{\partial \omega}\right|_{P=P^{*}, \omega=\omega^{*}}>0
$$


demand is less elastic w.r.t. price as advertising increases $\Rightarrow$ price and advertising are complements.

\section{Proof of Theorem 10}

Proof. The probability generating function of component sizes an individual with $k$ friends belongs to, conditional on not being in the giant component, is given by $\left(\frac{H_{1}(x, P)}{u}\right)^{k}$. The expected component size is $1+k \frac{H_{1}^{\prime}(1-\omega, P)}{u}\left(\frac{H_{1}(1-\omega, P)}{u}\right)^{k-1}=1+k \frac{H_{1}^{\prime}(1-\omega, P)}{u}$. Also the probability a person with $k$ friends is not in the giant component is $u^{k}$. Therefore

$$
k^{*}=\arg \max _{k \in\{0,1 \ldots\}}\left(1+k \frac{H_{1}^{\prime}(1-\omega, P)}{u}\right) u^{k}
$$

note that for $0<u<1 b>0$ the function $f(k)=(1+k b) u^{k}$ is continuous in $k$; has a maximum at $k^{* *}=\max _{k \geq 0}\left\{0,-\left(\frac{1}{\ln u(P)}+\frac{1}{b}\right)\right\}$ and $f^{\prime}(k)>0$ for $k<k^{* *}$ and $f^{\prime}(k)<0$ for $k>k^{* *}$. Hence $k^{*}$ is either the greatest integer below $\left\lfloor k^{* *}\right\rfloor$ or the smallest integer above $k^{* *},\left\lceil k^{* *}\right\rceil$. Thus

$$
k^{*} \in\left\{\left\lfloor k^{* *}\right\rfloor,\left\lceil k^{* *}\right\rceil\right\} \text { for } P<P^{c r i t}
$$

\section{Proof of Corollary 1}

Proof. We have

$$
\begin{aligned}
k^{* *} & =-\left(\frac{1}{\ln u(P)}+\frac{u(P)}{H_{1}^{\prime}(1-\omega, P)}\right) \\
& =-\left(\frac{1}{\ln u(P)}+\left(\frac{(1-P) G_{1}(u)}{u(P)\left(1-(1-P) G_{1}^{\prime}(u)\right)}\right)^{-1}\right) \\
& =-\left(\frac{1}{\ln u(P)}+\left(\frac{(1-P) \sum k p_{k} u^{k-2}}{\left(z_{1}-(1-P) \sum k(k-1) p_{k} u^{k-2}\right)}\right)^{-1}\right)
\end{aligned}
$$

where $\frac{1}{H_{1}^{\prime}\left(1-\omega, P^{\text {crit }}\right)}$ is finite so immediately $\lim _{P \rightarrow P^{\text {crit }}} \frac{-1}{\ln u(P)}=\infty \Rightarrow \lim _{P \rightarrow P^{\text {crit }}} k^{*}=\infty$. Also by definition $z_{1}-(1-P) \sum k(k-1) p_{k} u^{k-2}>0$ for $P<P^{\text {crit }} u>0$ and $(1-P) \sum k p_{k} u^{k-2}>0$ for $u(P)>0$ so $k^{*}$ is continuous in $u$ and hence $P$ for $P<P^{c r i t}$. Finally $\frac{(1-P) \sum k p_{k} u^{k-2}}{\left(z_{1}-(1-P) \sum k(k-1) p_{k} u^{k-2}\right)}>0$ so $-\frac{1}{\ln u(P)}$ is an upper bound on $k^{* *}$.

\section{Proof of Theorem 11}

Proof. Demand is given by

$$
D=\sum_{k} p_{k}\left(1-u^{k}\right) \int_{P}^{1} \Phi(\theta \mid k) d \theta
$$

where $u$ is the smallest non-negative solution to the self consistency condition:

$$
u=1-F_{1}(1)+F_{1}(u)
$$

The following lemma illustrates some properties of $u$ with respect to the price which I will subsequently use 
to prove the above theorem.

Lemma 2 Suppose $u(P)$ is given by the smallest non-negative solution to equation 6 and $q_{k}(P)$ is continuous in $P$ then

1. $u(P)=1$ and $\frac{d u}{d P}=0$ for $P^{\text {crit }} \leq P \leq 1$

2. $u<1$ and $u(P)$ is continuous in $P$ for $0 \leq P<P^{\text {crit }}$

3. Suppose further that $\sup _{k}\left|\frac{d q_{k}(P)}{d P}\right|<\infty$ for all $0 \leq P<P^{\text {crit }}$ then $\frac{d u}{d P}$ exists for all $0 \leq P<P^{\text {crit }}$

Proof. 1) $u(P)$ is the smallest non-negative solution to:

$$
\begin{aligned}
u & =1-F_{1}(1)+F_{1}(u) \\
& =1-\sum_{k} p_{k}^{\prime} q_{k}(P)+\sum_{k} p_{k}^{\prime} q_{k}(P) u^{k-1}
\end{aligned}
$$

let $\Upsilon(u, P)=1-\sum_{k} p_{k}^{\prime} q_{k}(P)+\sum_{k} p_{k}^{\prime} q_{k}(P) u^{k-1}$. Given the assumptions $\Upsilon(u, P)$ is continuous in both $P$ and $u$ and weakly decreasing and weakly increasing in each respectively. Further it is differentiable and strictly convex in $u$ for $u \in[0,1]$ provided $\sup _{k}\left|\frac{d q_{k}(P)}{d P}\right|<\infty$ and $q_{k}(P)>0$ for at least one $k>2$. If $P^{\text {crit }} \leq P<1$ then

$$
\sum_{k=2}^{\infty} p_{k}^{\prime}(k-1) q_{k}(P) \leq \sum_{k=2}^{\infty} p_{k}^{\prime}(k-1) q_{k}\left(P^{c r i t}\right)=1
$$

which implies that the smallest non-negative solution is $u=1$ since $\Upsilon(u, P)>u$ for all $u<1$ when $P^{\text {crit }} \leq P<1$.

2) Consider $u\left(P^{\prime}\right)$ and $u\left(P^{\prime \prime}\right)$ where $0<P^{\prime}<P^{\prime \prime}<P^{c r i t}$. Given that $q_{k}(P)$ is weakly decreasing and continuous in $P$ and $\Upsilon(u, P)$ is continuous, increasing and convex in $u$ then this implies $u\left(P^{\prime}\right)<u\left(P^{\prime \prime}\right)$. To prove continuity I will show that for any $\varepsilon>0$ such that $\left|u\left(P^{\prime}\right)-u\left(P^{\prime \prime}\right)\right|<\varepsilon$ then for $P \in\left(P^{\prime}, P^{\prime}+\delta\right)$ where $\delta=\frac{P^{\prime}-P^{\prime \prime}}{2}\left|u\left(P^{\prime}\right)-u(P)\right|<\varepsilon$. Given the definition of $\delta P^{\prime}<P<P^{\prime \prime}$. By the intermediate value theorem we have that $\Upsilon(u, P)-u=0$ has a solution in the interval $u \in\left[u\left(P^{\prime}\right), u\left(P^{\prime \prime}\right)\right]$. The continuity of $q_{k}(P)$ implies that the following is true $\Upsilon\left(u, P^{\prime}\right) \leq \Upsilon(u, P)$ and $\Upsilon\left(u, P^{\prime \prime}\right) \geq \Upsilon(u, P)$ for all $u$. Hence $\Upsilon\left(u(P), P^{\prime}\right)<u(P)<\Upsilon\left(u(P), P^{\prime \prime}\right)$ implying that $u\left(P^{\prime}\right)<u(P)<u\left(P^{\prime \prime}\right)$. We now have the result that $\left|u\left(P^{\prime}\right)-u(P)\right|<\varepsilon$.

3) To show differentiability consider the implicit relationship for $u: u-\Upsilon(u, P)=0$. Now differentiating

$$
\begin{aligned}
\frac{d u}{d P} & =\frac{\frac{d \Upsilon}{d P}}{1-\frac{d \Upsilon}{d u}} \\
& =\frac{\sum_{k} p_{k}^{\prime} \frac{d q_{k}(P)}{d P}\left(u^{k-1}-1\right)}{1-\sum_{k} p_{k}^{\prime}(k-1) q_{k}(P) u^{k-2}}
\end{aligned}
$$

the denominator is positive for $P<P^{c r i t}$ and the numerator exists provided $\sup _{k} \frac{d q_{k}(P)}{d P}<\infty$.

For $P<P^{\text {crit }}$

$$
\sum_{k=2}^{\infty} p_{k}^{\prime}(k-1) q_{k}(P)<\sum_{k=2}^{\infty} p_{k}^{\prime}(k-1) q_{k}\left(P^{c r i t}\right)=1
$$

the strictness of the inequality is given by the assumption that for any $\varepsilon>0 \exists k>2: q_{k}\left(P^{\text {crit }}\right)<$ $q_{k}\left(P^{c r i t}-\varepsilon\right)$. Hence $u(P)<1$ for $P<P^{c r i t}$. 
Using this lemma we now prove the parts of the main theorem.

1) $D(P)$ is given by

$$
D=\sum_{k} p_{k}\left(1-u^{k}\right) \int_{P}^{1} \Phi(\theta \mid k) d \theta
$$

which is continuous if $u(P)$ is continuous and $\Phi(\theta \mid k)$ contains no mass points which it does not by assumption.

2) The statement:

$$
\begin{aligned}
& D(P)=0 \text { for } P \geq P^{\text {crit }} \\
& D(P)>0 \text { for } P<P^{\text {crit }}
\end{aligned}
$$

follows from $u(P)=1$ for $P \geq P^{\text {crit }}$ and $u(P)<1$ for $P<P^{\text {crit }}$ in the earlier lemma and a positive fraction of individuals with $k \geq 1$ having valuations above the price.

3) $\frac{d D}{d P}$ is given by:

$$
\frac{d D}{d P}=-\frac{d u}{d P} \sum_{k} p_{k} k u^{k-1} \int_{P}^{1} \Phi(\theta \mid k) d \theta-\sum_{k} p_{k}\left(1-u^{k}\right) \Phi(\theta \mid k)
$$

which is 0 when $u(P)=1$ and exists and is decreasing for $P<P^{c r i t}$ since $\frac{d u}{d P} \geq 0$ and $\sum_{k} p_{k}\left(1-u^{k}\right) \Phi(\theta \mid k) d \theta>$ 0 for $u<1$.

4) When $\theta$ and $k$ are uncorrelated $\Phi(\theta \mid k)=1$ and $\frac{d u}{d P}>1$ for $P<P^{c r i t}$ and we have

$$
\begin{aligned}
\frac{d D}{d P} & =-\frac{d u}{d P} \sum_{k} p_{k} k u^{k-1}(1-P)-\sum_{k} p_{k}\left(1-u^{k}\right) \\
D(P) & =(1-P) \sum_{k} p_{k}\left(1-u^{k}\right)
\end{aligned}
$$

therefore

$$
\frac{P}{D} \frac{d D}{d P}=-\frac{P}{1-P}\left[\frac{\frac{d u}{d P} \sum_{k} p_{k} k u^{k-1}}{\sum_{k} p_{k}\left(1-u^{k}\right)}+1\right]
$$

where $\frac{\frac{d u}{d P} \sum_{k} p_{k} k u^{k-1}}{\sum_{k} p_{k}\left(1-u^{k}\right)}>0$.

\section{References}

[1] Cohen, Reuven, Keren Erez, Daniel Ben-Avraham, and Shlomo Havlin. 2000. "Resilence of the Internet to Random Breakdowns." Physical Review Letters 85(21): 4626-4628. 